\title{
A method to quantify and assess the dosimetric and clinical impact resulting from the heterogeneity correction in radiotherapy for lung cancer
}

\author{
Abdulhamid Chaikh1, 2, Jean-Yves Giraud1, 2, Jacques Balosso1, 2 \\ ${ }^{1}$ University Joseph Fourier, Grenoble, France. \\ ${ }^{2}$ Department of Radiation Oncology and Medical physics, Grenoble University Hospital, France.
}

Received January 03, 2014; Revised January 27, 2014; Accepted January 27, 2014; Published Online January 30, 2014

\section{Original Article}

\begin{abstract}
Purpose: The aim of this study was to propose a method to quantify and assess the differences in dose computations using heterogeneity correction algorithms for the planning target volumes and organs at risk. Methods: Six patients with lung cancer treated with 3-dimensional conformal radiation therapy were included and analysed. Dose calculations were performed using the pencil beam convolution (PBC) algorithm without heterogeneity correction and the Modified Batho method (PBC-MB) with heterogeneity correction. For each patient, 3 treatment plans were generated using exactly the same beam configuration. In plan 1, the dose was calculated using the PBC algorithm. In plan 2, the dose was calculated using the PBC-MB. In plan 3, the dose was calculated using the PBC-MB method but with the same number of monitor units obtained from plan 1 . To evaluate the treatment plans computed by the $\mathrm{PBC}$ and $\mathrm{PBC}-\mathrm{MB}$, the monitor units, dose at the isocenter, spatial isodose distribution, dose volume histograms, conformity index, homogeneity index, planning target volumes conformity index, and geometrical index were compared. A statistical analysis was carried out using Wilcoxon signed rank test. Results: The PBC-MB method in plan 2 produced a lower number of monitor units than in plan 1 using $\mathrm{PBC}$ algorithm $(\mathrm{p}<0.001)$. Dosimetric parameters derived from the dose volume histograms were higher for the planning target volumes and organs at risks using PBC-MB method for plans 2 and 3 when compared to plan 1 . There was no significant difference for all the quality indices between plan 1 and plan 2 , ( $p>0.05$ ), but a significant difference for the geometric index between plans 2 and $3(p=0.002)$ was observed. Conclusion: The risks related to the modification from the homogeneity plan to the heterogeneity plan were the reduction of delivered dose in monitor units for the planning target volumes and the increment of the dose to the organs at risk. We suggest the adaption in the dose prescriptions when switching the dose calculation algorithm from the PBC to PBC-MB.
\end{abstract}

Keywords: Heterogeneity Correction; Pencil Beam Convolution; Modified Batho Method

\section{Introduction}

Treatment planning is one of the main steps in radiotherapy, and it typically includes the isodose distributions and dose volume histograms (DVH) for the target volume and critical structures. The dose calculation in radiation therapy is performed using the algorithms employed in the treatment planning system (TPS). In a homogenous plan, the algorithms assume the patient has homogenous tissue density; whereas in a heterogeneous plan

Corresponding author: Abdulhamid Chaikh; Department of Radiation Oncology and Medical physics, Grenoble University Hospital, France. Email: abdulhamedc@yahoo.com

Cite this article as:

Chaikh A, Giraud J, Balosso J. A method to quantify and assess the dosimetric and clinical impact resulting from the heterogeneity correction in radiotherapy for lung cancer. Int J Cancer Ther Oncol 2014; 2(1):020110. DOI: 10.14319/ijcto.0201.10 the algorithm takes into account the different densities of the tissues. For clinical use, the algorithms must calculate the dose as accurately as possible. Several studies have evaluated the dose calculation algorithms for external photon beam therapy.1, 2, 3 They showed that the choice of algorithm could produce a large impact on the dose distribution. Additionally, a number of studies have assessed the impact of heterogeneity correction on dose calculations for lung cancer showing a large impact on the dose calculation. 4, 5, 6 It was concluded that the heterogeneity correction algorithms were not equivalent in their ability to calculate the dose for lung cancer patients. The aim of this study is to introduce a method for investigating the dosimetric and clinical impact of heterogeneity correction in lung cancer patients. We quantified and assessed the impact of the tissue heterogeneity correction in terms of monitor units (MUs), spatial isodose dis- 
tribution, and dosimetric parameters derived from cumulative DVH (cDVH). We also introduced the differential DVH (dDVH) and quality indices to evaluate the impact of heterogeneity correction on the dose distributions.

\section{Methods and Materials}

\section{Dose calculation algorithm}

Dose calculation in this study is based on pencil beam convolution (PBC) algorithm, which has been integrated in the Eclipse ${ }^{\circledR}$ TPS (Version 8.1; Varian Medical Systems, Palo Alto, CA). The PBC algorithm includes two calculation modes: without heterogeneity correction (hereafter referred as PBC), and with heterogeneity correction using a Modified Batho (hereafter referred as PBC-MB) method. Heterogeneity correction is typically based on the relative electron densities obtained from the computed tomography (CT)-Scan. The PBC-MB method employs an empirical correction factor that uses the tissue maximum ratios, and it takes into account the heterogeneity correction in 1D. The PBC algorithm considers the lateral scattering to calculate the dose for the homogeneous media. The heterogeneity correction is only applied in the longitudinal direction. The PBC-MB accounts the heterogeneity by using the equivalent path length converted from mass attenuation. The principle of PBC-MB is first to calculate a relative dose distribution within a medium of homogeneous water-equivalent composition, and then to add an inhomogeneity correction factor. This factor makes adjustments to the uncorrected distribution to account for variations in tissue density. $7,8,9,10,11$ All dose calculations in this study were done using a grid size was $2.5 \mathrm{~mm}$.

\section{Clinical cases}

This study is based on the dosimetric data of six patients treated for the lung cancer, which included a total of fourteen planning target volumes (PTVs). The patients were treated using the 3D radiotherapy irradiation technique with 18 mega-voltage (MV) photon beams. Table 1 shows the site locations, the number of PTVs, the prescribed dose, and the treatment fields for all six patients.

\section{Contouring}

For the treatment planning, the CT dataset of each clinical case were used. Physicians delineated the anatomic borders of target structures and organs at risk (OARs). The PTVs were defined according to the recommendations of ICRU Reports 50 and 62. ${ }^{12}$, 13 The PTVs were expanded from the clinical target volume with a margin to account for uncertainties in beam alignment, patient positioning, organ motion, and organ deformation. The virtual simulation for each patient was generated by a Digitally Reconstructed Radiograph (DRR) and Beam's Eye View (BEV) information.

\section{Treatment planning}

For each patient, 3 treatment plans were generated using exactly the same configuration of beams, collimator, and accessories. In plan 1, the dose was calculated using the PBC without the heterogeneity correction. In plan 2, the dose was calculated using the PBC-MB method for the heterogeneity correction. In plan 3, the dose was calculated using the PBC-MB method but with the same number of MUs as obtained from plan 1. In all plans, the dose was prescribed at a single reference point as recommended by the ICRU Reports 50 and 62. ${ }^{12,13}$ The reference treatment plans were designed according to the clinical experience of the department and ICRU recommendations. ${ }^{12,13}$ Figure 1 shows the CT-Scan for the parenchyma site (Patient \# 1). The red and yellow circles show the PTV2 with a field size of $5.4 \times 5.3 \mathrm{~cm}^{2}$ and the PTV1 with a field size of $6.4 \times 6.4 \mathrm{~cm}^{2}$, respectively. In this case, the patient was treated using 6 treatment fields. Specifically, each PTV was treated using three fields: one lateral and two oblique.

For each patient, to compare plan 2 with plan 1, the calculated dose in plan 1 was taken as the reference value. The percentage difference was calculated as:

$$
\Delta \text { Dose }(\%)=\left(\frac{\mathrm{D} 2-\mathrm{D} 1}{\mathrm{D} 1}\right) \times 100
$$

For each patient, to compare plan 3 with plan 2, the calculated dose in plan 2 was taken as the reference value. The percentage difference was calculated as:

$$
\Delta \text { Dose }(\%)=\left(\frac{\mathrm{D} 3-\mathrm{D} 2}{\mathrm{D} 2}\right) \times 100
$$

\section{Dosimetric analysis}

\section{MUs and isocenter dose}

For each field in a given patient case, the MUs comparison was done between the plan 1 and plan2 for each field. The isocenter dose calculated by the $\mathrm{PBC}-\mathrm{MB}$ in plan 3 was compared to that of prescribed dose in plan 1 .

\section{Spatial isodose distribution}

The isodose curves at 40\%, 95\%, and $100 \%$ were compared plans 1,2 , and 3 .

\section{$\mathrm{DVH}$}

For each PTV, the maximum dose, minimum dose, mean dose, dose to $95 \%$ of the PTV volume (D95), and the volume of PTV receiving at least $95 \%$ of the prescribed dose (V95) were compared using $\mathrm{cDVH}$. The $\mathrm{dDVH}$ was used to compare the dose homogeneity inside the PTV using the standard deviation associated with the dDVH. For the normal lung, (i.e., lung - PTV), the maximum dose, minimum dose and mean dose were compared. Additionally, the comparison was done for the volume fractions of normal lung receiving 10, 13, 20, and 30 Gy (V10, V13, V20, and V30 Gy, respectively). For the spinal cord, the maximum dose was compared. 
TABLE 1: Characteristics of the 6 patients treated with 3D radiotherapy using a 18 MV photon beam, tumour location, volume of PTVs, dose prescription, and treatment fields. The PTVs number shows the multiple PTVs for the same case.

\begin{tabular}{|c|c|c|c|c|c|}
\hline Patients & Locations & PTVs & $\begin{array}{c}\text { PTV } \\
\text { Volumes }\left[\mathrm{cm}^{3}\right]\end{array}$ & $\begin{array}{c}\text { Dose prescription } \\
{[\mathrm{Gy}]}\end{array}$ & Number of Treatment fields \\
\hline \multirow[t]{2}{*}{1} & Right parenchyma & 1 & 75 & 50 & 3 \\
\hline & & 2 & 38 & 16 & 3 \\
\hline \multirow[t]{3}{*}{2} & Left retro cardiac & 1 & 618 & 50 & 9 \\
\hline & & 2 & 329 & 10 & 4 \\
\hline & & 3 & 150 & 6 & 4 \\
\hline \multirow[t]{3}{*}{3} & Top left lung & 1 & 343 & 50 & 4 \\
\hline & & 2 & 207 & 14 & 3 \\
\hline & & 3 & 102 & 6 & 3 \\
\hline \multirow[t]{2}{*}{4} & Mediastina & 1 & 332 & 50 & 5 \\
\hline & & 2 & 115 & 10 & 4 \\
\hline \multirow[t]{2}{*}{5} & Mediastina & 1 & 616 & 50 & 8 \\
\hline & & 2 & 295 & 10 & 4 \\
\hline \multirow[t]{2}{*}{6} & Oesophagus & 1 & 1136 & 45 & 4 \\
\hline & & 2 & 554 & 9 & 4 \\
\hline
\end{tabular}

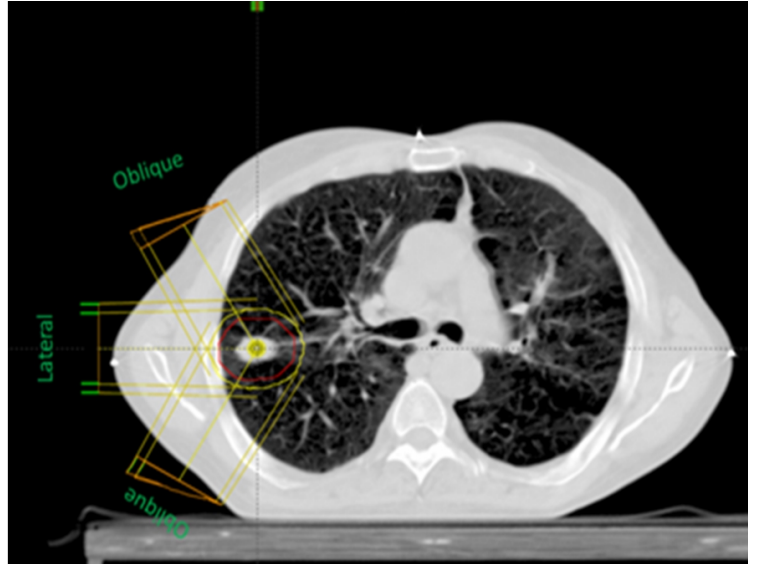

FIG. 1: CT-Scan for the parenchyma site (Patient \# 1). The red and yellow circles show PTV2 with a field size $5.4 \times 5.3 \mathrm{~cm}^{2}$ and PTV1 with a field size $6.4 \times 6.4 \mathrm{~cm}^{2}$, respectively.

\section{Quality indices}

The following quality indices were calculated and compared. ${ }^{14,15}$ :

The Conformity Index (CI) was used to compare the plan conformity, defined as:

$$
\mathrm{CI}=\frac{\text { Minimum dose encompassing the PTV }}{\text { Prescribed Dose }}
$$

The Homogeneity Index (HI) was used to compare the dose homogeneity in the PTV, defined as:

$$
\mathrm{HI}=\frac{\text { Maximum Dose in the PTV }}{\text { Prescribed Dose }}
$$

The PTV Conformity Index (CIPTV) was used to compare the degree of conformity of the prescribed dose, defined as:

$$
\text { CIPTV }=\frac{\text { V95 }}{\text { PTV volume }}
$$

where, V95 is the PTV volume receiving more than $95 \%$ of the prescribed dose.

The geometrical index (g) was used to compare the geometric conformity to the PTV and normal tissues, defined as:

$$
g=\frac{\mathrm{VPTV}+\mathrm{VNT}}{\mathrm{PTV} \text { volume }}
$$

where VPTV designates the PTV volumes receiving a dose lower than $100 \%$ of the prescribed dose and VNT are the normal tissue volumes receiving $100 \%$ of the prescribed dose.

\section{Statistical analysis}

The Wilcoxon signed rank test was used to calculate the $p$-value using an alpha error at 5\%. Data are presented as average \pm standard deviation (SD).

\section{Results}

\section{MUs and isocenter dose}

The PBC-MB in plan 2 calculated significantly less MUs than the $\mathrm{PBC}$ in plan 1 by on average $5.2 \%$ (4.4 SD; $p<0.001$ ). For the dose at the isocenter, the $\mathrm{PBC}-\mathrm{MB}$ calculated a higher dose in plan 3 than the prescribed dose in plan 1 by on average $5.2 \%(1.6 \mathrm{SD} ; p=0.002)$. 


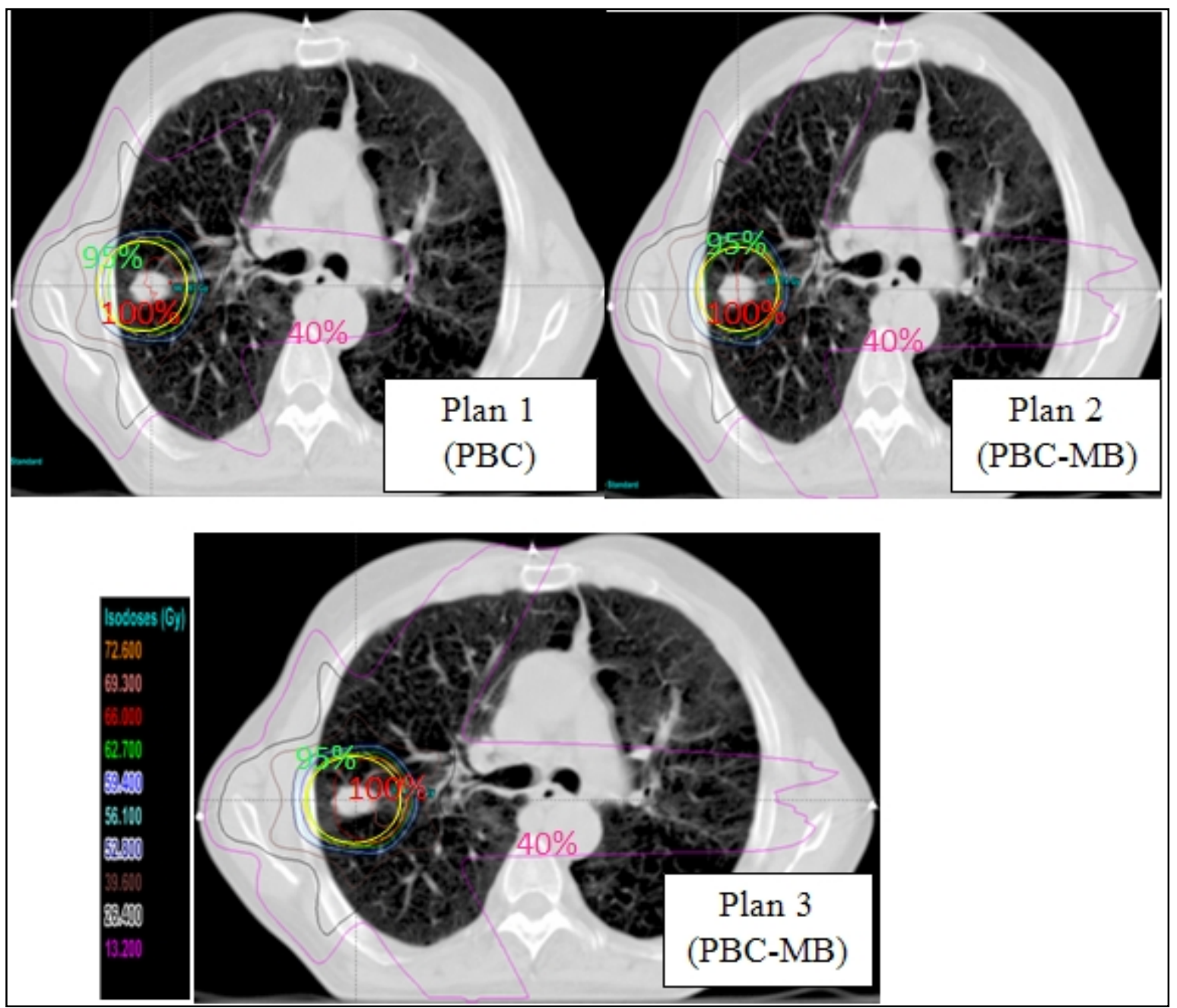

FIG. 2: Transverse views of isodose distribution curves for plan 1 without heterogeneity correction using PBC, and plans 2 and 3 with heterogeneity corrections using $\mathrm{PBC}-\mathrm{MB}$. A dose of 66Gy was prescribed at isocenter for plan 1 and plan 2 . The dose in plan 3 was recalculated using $\mathrm{PBC}-\mathrm{MB}$ with the same number of monitor units as in the PBC, and the prescribed dose at isocenter was 68Gy. The yellow contour shows the PTV, whereas the red, green, and orange colours represent the $100 \%, 95 \%$, and $40 \%$ isodose curves, respectively.

\section{Spatial Isodose distribution}

Figure 2 shows the transverse views of isodose distribution curves for plan 1 (PBC), and plan 2 and plan 3 (PBC-MB). In figure 2, it can be seen that the $95 \%$ isodose curves in all three plans included the whole PTVs. The 100\% isodose curves enclosed the PTVs using plan 2 and plan 3 to a greater extent than in plan 1 . The $40 \%$ isodose curves calculated by PBC-MB in plan 2 and plan 3 encompassed a greater volume of normal lung tissue when compared with that of plan 1 .

\section{DVH}

Figures 3 and 4 show the $\mathrm{cDVH}$ and $\mathrm{dDVH}$, rspectively, for a parenchyma site using the $\mathrm{PBC}$ without heterogeneity correction in plan 1, and the PBC-MB for plans 2 and 3. Tables 2 and 3 summarize the dosimetric and statistical results for the PTVs and normal lung and the results were averaged for all 6 cases. In tables 2 and 3, it can be seen that the PBC-MB in plans 2 and 3 calculated a higher dose for the PTVs and normal lung compared to plan 1. For the normal lung, all three plans showed the results of V20 and V30 within the tolerance. For the spinal cord, the maximum dose in plans 2 and 3 was higher by an average of 5\% (12 SD) with no statistical significance $(p=0.485)$. The dose constraint for spinal cord (maximum dose $\leq 45 \mathrm{~Gy}$ ) was respected in all plans.

The comparison of dDVH showed that the PBC-MB calculated a heterogeneous dose in plan 2 and plan 3 compared with $\mathrm{PBC}$ in plan 1 . The averages of the deviation standard associated with dDVH were $3.2 \mathrm{~Gy}(2.1 \mathrm{SD}), 3.6 \mathrm{~Gy}(2.4 \mathrm{SD})$, and $3.7 \mathrm{~Gy}(2.2 \mathrm{SD})$ for plan 1, plan 2 and plan 3 , respectively. There was a significant difference between the dDVH calculated in plan 1 and plan $2(p=0.012)$, but there was no significant difference between the dDVH calculated in plan 2 and plan $3(\mathrm{p}=0.055)$. 


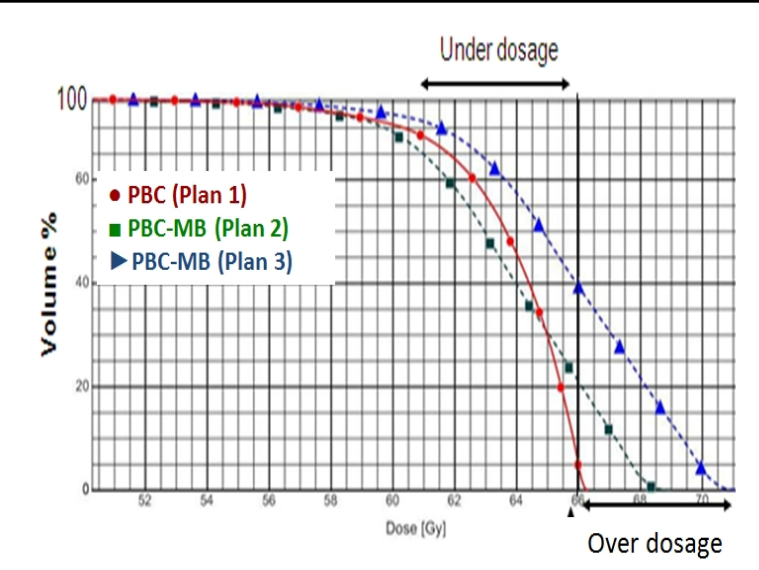

FIG. 3: Cumulative dose volume histograms for patient 1 . The histograms were calculated using the $\mathrm{PBC}$ algorithm without tissue heterogeneity correction for plan 1 and the $\mathrm{PBC}-\mathrm{MB}$ method (with heterogeneity correction) for plans 2 and 3 . The dose in plan 3 was calculated using $\mathrm{PBC}-\mathrm{MB}$ for the same number of monitor units as in the PBC for plan 1. The prescription dose for plan 1 and 2 was $66 \mathrm{~Gy}$, but it was $68 \mathrm{~Gy}$ for plan 3 .

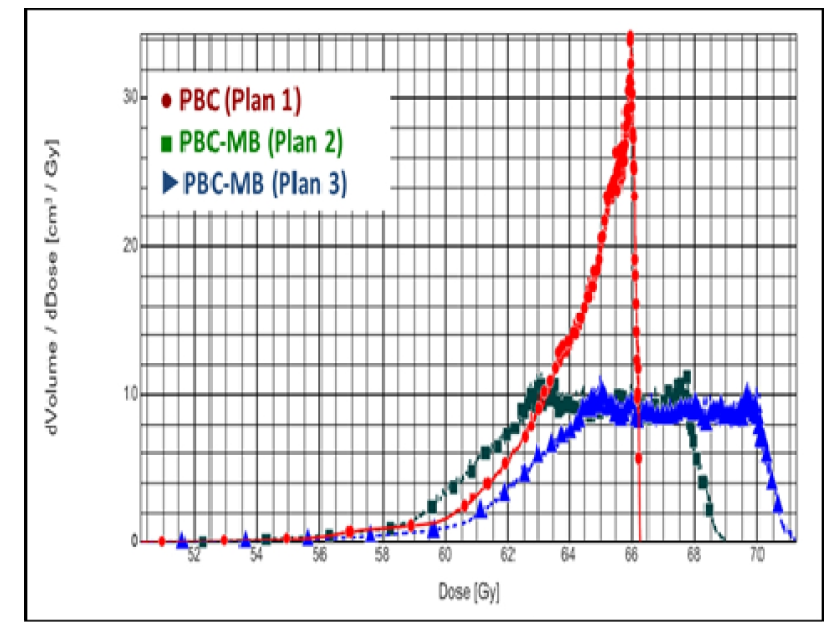

FIG. 4: Differential dose volume histograms for patient 1 . The histograms were calculated using the $\mathrm{PBC}$ algorithm without heterogeneity correction for plan 1 and the PBC-MB method with heterogeneity correction for plans 2 and 3 . The dose in plan 3 was calculated using $\mathrm{PBC}-\mathrm{MB}$ for the same number of monitor units as in the $\mathrm{PBC}$ for plan 1 . The prescription dose for plan 1 and 2 was $66 \mathrm{~Gy}$, but it was 68 Gy for plan 3.

TABLE 2: Dose-volume parameters derived DHVs for the planning target volume (PTV). The results are averaged over all 6 analyzed cases.

\begin{tabular}{ccccccc}
\hline \hline Plans & $\Delta$ Dose $\%$ & Mean & Minimum & Maximum & D95 & V95 \\
\hline 2 vs. 1 & average \pm SD & $0.4 \pm 1$ & $0.9 \pm 3.5$ & $1.9 \pm 1.4$ & $1.6 \pm 1.8$ & $2.4 \pm 4.7$ \\
& $p$-value & 0.06 & 0.04 & 0.01 & 0.003 & 0.03 \\
\hline 3 vs. 2 & average \pm SD & $3.8 \pm 2.5$ & $3.9 \pm 2.4$ & $2.7 \pm 2.7$ & $4.6 \pm 1.9$ & $8 \pm 8$ \\
& $p$-value & 0.005 & 0.001 & 0.02 & 0.001 & $<0.001$ \\
\hline \hline
\end{tabular}

$\Delta$ is the difference of values between plan 2 and plan 1 or plan 3 and plan $2 . \Delta \mathrm{Dose} \%$ was calculated according to equations 1 and $2 . \mathrm{SD}$ is the standard deviation. The D95 is the calculated dose to 95\% of the PTV volume. The V95 is the volume of PTV receiving at least $95 \%$ of the prescribed dose. The p-values were calculated using a Wilcoxon signed rank test. The p-value of less than 0.05 was considered to be statistically significant.

TABLE 3: Dose volume parameters deriving from dose volume histograms for normal lung tissue (i.e., lung - PTV). The results are averaged over all 6 analyzed cases.

\begin{tabular}{|c|c|c|c|c|c|c|c|c|}
\hline Plans & $\Delta$ Dose $\%$ & Mean & Minimum & Maximum & V10 & V13 & V20 & V30 \\
\hline \multirow[t]{2}{*}{2 vs. 1} & average \pm SD & $8.8 \pm 14$ & $1 \pm 5.6$ & $1.5 \pm 2$ & $4.1 \pm 6.2$ & $7.6 \pm 17.8$ & $0.5 \pm 12.8$ & $0.4 \pm 5.2$ \\
\hline & $p$-value & 0.1 & 0.1 & 0.03 & 0.02 & 0.1 & 0.3 & 0.4 \\
\hline \multirow[t]{2}{*}{3 vs. 2} & average $\pm \mathrm{SD}$ & $5.7 \pm 3 . \overline{6}$ & $5.5 \pm 3.3$ & $0.8 \pm 9.8$ & $2.5 \pm 4.7$ & $4.4 \pm 5.7$ & $33 \pm 47.1$ & $4.6 \pm 3.8$ \\
\hline & $p$-value & 0.001 & 0.03 & 0.2 & 0.1 & 0.03 & 0.001 & 0.03 \\
\hline
\end{tabular}

$\Delta$ is the difference of values between plan 2 and plan 1 or plan 3 and plan $2 . \Delta$ Dose\% was calculated according to equations 1 and 2 . SD is the standard deviation. The V10, V13, V20, and V30 Gy are defined as the volume fractions of normal lung receiving 10, 13, 20, and 30 Gy, respectively. The p-values were calculated using a Wilcoxon signed rank test. The p-value of less than 0.05 was considered to be statistically significant.

\section{Quality indices}

Table 4 summarizes the quality indices for all the PTVs in plan 1 , plan 2 , and plan 3 . The comparison between plan 1 and plan 2 showed that there was no statistically significant difference for all the indices, $(p>0.05)$. The comparison between plan 2 and plan 3 showed a significant difference for the geometric indices $(p=0.002)$. 
TABLE 4: Quality indices in plans 1, 2, and 3. Data are presented as average \pm standard deviation. Furthermore, results provided below are averaged over all 6 analyzed cases.

\begin{tabular}{ccccc}
\hline \hline Plans & CI & HI & CIPTV & g \\
\hline Plan 1 & $0.8 \pm 0.2$ & $1 \pm 0.07$ & $0.8 \pm 0.2$ & $0.2 \pm 0.2$ \\
Plan 2 & $0.9 \pm 0.1$ & $1 \pm 0.05$ & $0.7 \pm 0.2$ & $0.2 \pm 0.2$ \\
$p$-value (2 vs 1$)$ & $>0.05$ & $>0.05$ & $>0.05$ & $>0.05$ \\
\hline Plan 3 & $0.8 \pm 0.1$ & $1.1 \pm 0.1$ & $0.8 \pm 0.2$ & $0.2 \pm 0.2$ \\
$p$-value (3 vs 2 ) & $>0.05$ & $>0.05$ & $>0.05$ & 0.002
\end{tabular}

$\mathrm{CI}$ is the Conformity Index. HI is the Homogeneity Index. CIPTV is the Conformity Index for the planning target volumes. $\mathrm{g}$ is the geometrical index. The p-values were calculated using a Wilcoxon signed rank test. The p-value of less than 0.05 was considered to be statistically significant.

\section{Discussion}

There are a wide variety of dose calculation algorithms commercially available for the heterogeneity corrections in radiation therapy. ${ }^{3,16}$ Several studies have investigated the change from $\mathrm{PBC}$ to Anisotropic Analytical Algorithm (AAA) for Eclipse. 6, 17, 18, 19, 20, 21 It has been shown that the PBC algorithm employed with Eclipse TPS could produce errors in dose estimation. Thus, it has been suggested that more accurate calculation methods are used in clinical radiotherapy planning. In particular, Rana recommended using more accurate dose calculation in the treatment planning for the lung cancer. ${ }^{22}$ In our study, we tackle the problem of changing the calculation algorithms from the $\mathrm{PBC}$ without heterogeneity correction to the $\mathrm{PBC}$ with heterogeneity correction. In order to offer clinicians with treatment plans that are much closer to reality than the plans generated with the PBC without heterogeneity correction, we posed the question as to whether the clinician should adapt the dose prescriptions. Normally, these prescriptions are the result of decades of clinical experience and are based on older and less accurate methods of dose calculation. We strongly suggest that a sufficient number of cases should be calculated for each anatomical site with the old and the new algorithms. This is necessary, because we observed that the differences in calculated dose vary from patient to patient. Hence, we propose clinicians some recommendations to assist adaption of the prescribed dose when using new calculation methods.

In this study, the PBC-MB was used to study the impact of tissue heterogeneity correction in the lung plans. However, the PBC-MB is not an accurate correction method since it does not take into account the changes in lateral electron transport. The tissue densities introduced a difference between the PBC without heterogeneity correction and PBC-MB with heterogeneity correction. In fact, the lung exhibits the most heterogeneous anatomy with bone, lung, and soft tissues. A majority of lateral and oblique beams pass through the low density tissue of the lung. For this reason, the heterogeneity correction showed a dosimetric impact on PTVs and normal lung tissue. The comparison between plan 1 and plan 2 showed that the number of MUs was significantly higher in plan 1 compared to plan 2 using $18 \mathrm{MV}$ photon beams. A comparison of plan 1 and plan 3, which used the same number of MUs, showed that the calculated dose was significantly higher in plan 3 compared to plan 1. The difference in MUs depends on the site location, PTVs volumes, and field size. This means that the geometric configuration or the dose weight of beams must be modified to respect the dose constraints for the normal lung tissues. We recognize that a limitation of the present study was the small population number $(n=6)$.

In practice, clinical dose distributions are not uniform for the PTVs due to variations in tissue density. The comparison of dDVH showed that the PBC algorithm without density correction produced a narrower differential histogram and a larger number of volume fractions of the PTVs receiving the prescribed dose than for PBC-MB. This means that PBC without heterogeneity correction tends to give a better PTV coverage. Nevertheless, the method with the heterogeneity correction is closer to the reality. ${ }^{16}$ The use of the PBC-MB produced under and over estimations of dose for some areas of the PTVs and hot spots in normal tissue outside the PTVs.

Our results show that the quality indices provide a qualitative comparison between treatment plans and quantify the degree of congruence between the isodose and the prescribed dose. The standard deviation associated with the $\mathrm{dDVH}$ provides a more reliable and accurate measure of dose homogeneity within the PTVs than the HI. For example, the standard deviations associated with the $\mathrm{dDVH}$ were $2.2 \mathrm{~Gy}, 2.9 \mathrm{~Gy}$ and $3 \mathrm{~Gy}$ for plan 1, plan 2 and plan 3 respectively. While HI values were equal to unity using plan 1 and plan 2, it was 1.1 using plan 3 . We recommend improving the treatment ballistic to take advantage of the heterogeneity correction methods for the protection of the OARs by adjustment of target dose prescription, dose constraints, and evaluation of the tumour control probability (TCP) and the normal tissue complication probability (NTCP). Radiobiological treatment plan evaluation of lung plans could potentially provide more accurate values for the TCP and NTCP. ${ }^{23}$ 


\section{Conclusion}

The method presented in this study enables clinicians and physicians to understand treatment modifications associated with changes in dose calculation software. Tissue heterogeneity correction had a significant impact on dose distribution for the PTVs and OARs. The number of MUs significantly decreased using heterogeneity correction PBC-MB. These results should be discussed among clinicians and medical physicists to decide whether or not an alteration of the current prescription procedures and plan acceptability criteria should be considered.

\section{Conflict of interest}

The authors declare that they have no conflicts of interest. The authors alone are responsible for the content and writing of the paper.

\section{References}

1. Morgan AM, Knoos T, McNee SG, Evans CJ, Thwaites DI. Clinical implications of the implementation of advanced treatment planning algorithms for thoracic treatments. Radiother Oncol 2008; 86: 4854.

2. Knoos T, Wieslander E, Cozzi L, Brink C, Fogliata A, Albers D, Nystrom H, Lassen S. Comparison of dose calculation algorithms for treatment planning in external photon beam therapy for clinical situations. Phys Med Biol 2006; 51: 5785-5807.

3. Task Group No. 65, the Radiation Therapy Committee of the American Association of Physicists in Medicine. Tissue inhomogeneity corrections for MV photon beams. Madison, WI: Medical Physics Publishing 2004.

4. Gray A, Oliver LD, Johnston PN. The accuracy of the pencil beam convolution and anisotropic analytical algorithms in predicting the dose effects due to attenuation from immobilization devices and large air gaps. Med Phys 2009; 36: 3181-91.

5. Rana S, Pokharel S. Verification of dose calculation algorithms in a multi-layer heterogeneous phantom using films. Gulf J Oncolog 2013; 1:63-9.

6. Narabayashi M, Mizowaki T, Matsuo Y, Nakamura M, Takayama K, Norihisa Y, Sakanaka K, Hiraoka M. Dosimetric evaluation of the impacts of different heterogeneity correction algorithms on target doses in stereotactic body radiation therapy for lung tumors. JRadiat Res 2012; 53: 777-84.

7. Ahnesjo A, Aspradakis MM. Dose calculations for external photon beams in radiotherapy. Phys Med Biol 1999; 44: R99-155.

8. Batho HF. Lung corrections in cobalt 60 beam therapy. J Can Assoc Radiol 1964; 15: 79-83.

9. El-Khatib E, Battista JJ. Improved lung dose calculation using tissue-maximum ratios in the Batho correction. Med Phys1984; 11: 279-286.
10. Thomas SJ. A modified power-law formula for inhomogeneity corrections in beams of high-energy $\mathrm{x}$ rays. Med Phys 1991; 18: 719 -723.

11. Lu L. Dose calculation algorithms in external beam photon radiation therapy. Int $J$ Cancer Ther Oncol 2013; 1: 01025.

12. ICRU Report No. 50. Prescribing, Recording and Reporting Photon Beam Therapy.International Commission on Radiation Units and Measurements, Bethesda, Maryland 1993.

13. ICRU Report No. 62. Prescribing, Recording and Reporting Photon Beam Therapy supplement to ICRU Report 50, International Commission on Radiation Units and Measurements, Bethesda, Maryland 1999.

14. Feuvret L, Noel G, Nauraye C, Garcia P, J-Mazeron J. Conformal index and radiotherapy. Cancer Radiother 2004; 8: 108-119.

15. Lomax NJ, Scheib SG. Quantifying the degree of conformity in radiosurgery treatment planning. Int $J$ Radiat Oncol Biol Phys 2003; 55:1409-19.

16. Wong JW, Purdy JA. On methods of inhomogeneity corrections for photon transport. Med Phys 1990; 17: 807-14.

17. Hedin E, Back A. Influence of different dose calculation algorithms on the estimate of NTCP for lung complications. J Appl Clin Med Phys 2013; 14: 127-39.

18. Seppala J, Suilamo S, Kulmala J, Mali P, Minn H. A dosimetric phantom study of dose accuracy and build-up effects using IMRT and RapidArc in stereotactic irradiation of lung tumours. Radiat Oncol 2012; 7: 79.

19. Herman Tde L, Hibbitts K, Herman T, Ahmad S. Evaluation of pencil beam convolution and anisotropic analytical algorithms in stereotactic lung irradiation. JMed Phys 2011; 36: 234-238.

20. Rana S, Rogers K, Pokharel S, Cheng C. Evaluation of Acuros XB algorithm based on RTOG 0813 dosimetric criteria for SBRT lung treatment with RapidArc. $J$ Appl Clin Med Phys 2014; 15: 4474.

21. Herman Tde L, Gabrish H, Herman TS, Vlachaki MT, Ahmad S. Impact of tissue heterogeneity corrections in stereotactic body radiation therapy treatment plans for lung cancer. J Med Phys 2010; 35: 170-173.

22. Rana S. Clinical dosimetric impact of Acuros $X B$ and analytical anisotropic algorithm (AAA) on real lung cancer treatment plans: review. Int $J$ Cancer Ther Oncol 2014; 2: 02019.

23. Mavroidis P. Clinical implementation of radiobiological measures in treatment planning. Why has it taken so long? Int J Cancer Ther Oncol 2013; 1:01019. 